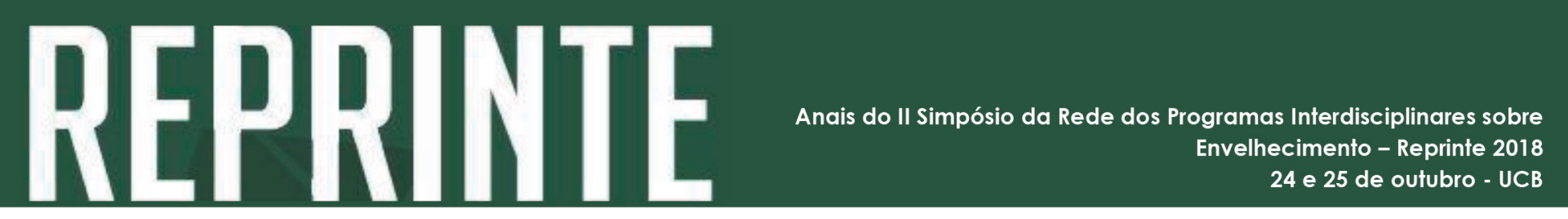

http://dx.doi.org/10.5335/rbceh.v16i1.9799

\title{
7) Estado nutricional de portadores de transtornos mentais internados em residências terapêuticas
}

\author{
Paula Manfredi'; Ana Luisa Sant' Anna Alves²; Ana Carolina Bertoletti De Marchi²; \\ Bernadete Maria Dalmolin²; Miriam Mattos²
}

\section{Resumo}

No Brasil, um rápido envelhecimento populacional é observado. Algumas populações possuem características diferentes da população em geral, como taxa de mortalidade, estado nutricional e uso de medicamentos, que é o caso de indivíduos com transtornos mentais. O objetivo deste estudo foi descrever o estado nutricional de indivíduos em tratamento em Residenciais Terapêuticos (RT). Tratase de um estudo transversal com indivíduos com transtornos mentais em tratamento em RT no município de Passo Fundo/RS. Foram entrevistados 68 usuários-moradores e destes, 39,6\% ( $n=17)$ apresentaram excesso de peso e $30,2 \%(n=13)$ obesidade. O uso excessivo de medicamentos surge como um fator determinante para o estado nutricional alterado, visto que todos os indivíduos usam pelo menos um medicamento contínuo e sugerem ter relação com o aumento de peso.

Palavras-chave: Institucionalização. Transtornos mentais. Estado nutricional. Tratamento.

\section{Introdução}

O rápido envelhecimento populacional é influenciado pela transição demográfica atual, processo que envolve a diminuição das taxas de fecundidade e natalidade e aumento da expectativa de vida, e, associada à transição demográfica, observa-se a transição epidemiológica, marcada pela mudança no padrão de adoecimento (LEBRÃO, 2007).

O processo de envelhecimento em indivíduos com transtornos mentais e comportamentais pode ser agravado, pois muitos são institucionalizados precocemente, fazem uso excessivo de medicamentos, especialmente psicotrópicos, e apresentam estado nutricional comprometido. Tais fatos podem contribuir para diminuição da expectativa de vida. Assim, por se tratar

1 Nutricionista e mestranda em Envelhecimento Humano, Faculdade de Educação Física e Fisioterapia, Universidade de Passo Fundo. Endereço para correspondência: Universidade de Passo Fundo, Campus I, BR 285, Bairro São José, CEP 99052-900, Passo Fundo, Rio Grande do Sul, Brasil Email: paula.manfredi@hotmail.com

2 Professores do Programa de Pós-Graduação Stricto Sensu em Envelhecimento Humano, Faculdade de Educação Física e Fisioterapia, Universidade de Passo Fundo. Emails (na ordem em que aparecem): alves.als@upf.br; carolina@ upf.br; berna@upf.br; miriam@upf.br 
de um agravo com curso muito variável, surge a necessidade de compreender melhor as suas repercussões ao longo do processo de envelhecimento (GONÇALVES et al, 2001).

Atualmente, os Serviços Residenciais Terapêuticos (SRT) surgem como casas destinadas a responder a necessidade de moradia de pessoas com transtornos mentais e comportamentais, egressas de hospitais psiquiátricos ou não. Este dispositivo residencial pode servir também de apoio a usuários de outros serviços em Saúde Mental, como os dependentes de álcool e outras drogas que não possuam suporte familiar e social. No entanto, trata-se de um processo de asilamento, excluindo-os dos seus contextos familiares e sociais (MINISTÉRIO DA SAÚDE, 2005).

Pensando na qualidade e expectativa de vida de indivíduos com transtornos mentais e comportamentais institucionalizados, com vista à promoção da saúde e prevenção de doenças, o objetivo desse estudo foi descrever o estado nutricional de indivíduos em tratamento de Residenciais Terapêuticos.

\section{Portadores de transtornos mentais e comportamentais}

Alguns grupos populacionais apresentam taxa de mortalidade maior que a taxa da população em geral, devido a presença de doenças e agravos. Portadores de transtornos mentais e comportamentais em comparação aos não portadores apresentam expectativa de vida $20 \%$ menor (SAMPAIO et al, 2016).

Entre diversas doenças mentais, a maior prevalência é de esquizofrenia e transtornos delirantes, diagnósticos identificados através de um estudo realizado em residenciais terapêuticos de Recife, em Pernambuco (FRANÇA, 2017).

Em outro estudo, realizado em um centro de atenção psicossocial de Porto Alegre (RS), a patologia mais frequente foi também a esquizofrenia, porém transtornos de humor e transtornos de personalidade também apareceram (KENGERISKI et al, 2015).

Ainda, um estudo realizado no estado do Ceará mostrou uma maior prevalência, mais uma vez, de esquizofrenia entre os pacientes institucionalizados. Além disso, outros diagnósticos apareceram, como psicose não orgnânica não especificada, retardo mental, demência e outros (MELO et al, 2015).

No que diz respeito ao estado nutricional, indivíduos com transtornos mentais possuem um estado nutricional diferente comparados à indivíduos normais, principalmente devido ao uso de psicofármacos. Alguns estudos sugerem que o uso de medicação antipsicotica seja um fator associado a alta frequência de sobrepeso e obesidade dessa população. A prevalência de obesidade na população de pacientes com esquizofrenia tratados com antipsicoticos varia entre $40 \%$ e $60 \%$ (WIRSHING, 2004).

Em uma unidade de saúde em Fortaleza foi realizado um estudo que avaliou o estado nutricional de 146 indivíduos em uso de antipsicotico. Pela classificação do IMC, 25,34\% $(n=37)$ apresentavam sobrepeso e 28,08\% ( $n=41)$ obesidade (SAMPAIO et al, 2016).

Outro estudo, realizado com 25 pacientes esquizofrênicos atendidos no Hospital de Clínicas de Porto Alegre, revelou uma frequência de 40\% de sobrepeso e 40\% de obesidade, conforme verificado pelo IMC. Entretanto estes pacientes estavam em uso de antipsicóticos (NUNES et al, 2014). 
Também foi encontrado como resultado de outro estudo, realizado com indivíduos com transtornos mentais de residenciais terapêuticos, em uso de medicação, em Alfenas, Minas Gerais, maior prevalência de sobrepeso (61\%), através do IMC (GARCIA et al, 2013).

Além disso, o estado nutricional comprometido leva à diminuição da capacidade funcional e qualidade de vida, bem como aumento do risco de morbidade e mortalidade (JOHANSSON et al, 2017).

Para prevenção e controle do ganho de peso, o acompanhamento nutricional e a realização de medidas antropométricas, associados com o estilo de vida e nível de atividade física regular, são recomendados e auxiliam na qualidade de vida dessa população, ainda mais se a medicação utilizada indicar o aumento de peso (LEITÃO-AZEVEDO et al, 2007). O excesso de peso nem sempre é dado somente como uma reação adversa da medicação, e sim pela má nutrição e inatividade física (REIS et al, 2007).

\section{Materiais e métodos}

Trata-se de um estudo transversal com indivíduos com transtornos mentais em Residenciais Terapêuticos Privados (RTP) registrados na Vigilância em Saúde da Secretaria Municipal de Saúde de Passo Fundo.

Este estudo faz parte do projeto "Censo sociodemográfico, jurídico e de saúde dos usuáriosmoradores das Instituições de Longa Permanência Terapêutica de Passo Fundo/RS", que foi aprovado pelo Comitê de Ética em Pesquisa da Universidade de Passo Fundo sob parecer n 2.092.340 e todos os indivíduos estão sendo preservados através do Termo de Consentimento Livre e esclarecido.

Para responder os objetivos do presente estudo foi aplicado questionário com as características demográficas e socioeconômicas. Tais variáveis foram coletadas dos prontuários. A variável sexo será categorizada em feminino e masculino, a idade será calculada a partir da data de nascimento e momento da entrevista e codificada em anos completos, o estado civil codificado em solteiro, casado/união estável, viúvo e separado/divorciado e cor da pele será codificada em branco, pardo, preto, indígena, amarelo e informação ignorada.

O estado nutricional foi avaliado do através do Índice de Massa Corporal (IMC), calculado peso $(\mathrm{kg})$ / altura (m) x altura (m), classificado como <18,5 magreza, 18,6 - 24,9 eutrofia, 25,0 - 29,9 sobrepeso, 30,0-34,9 Obesidade grau I, 35,0 - 39,9 obesidade grau II, > 40,0 obesidade grau III (OMS, 1995).

Os dados foram digitados e analisados em software de estatística. Calculou-se as medidas de tendência central e dispersão para as variáveis quantitativas e apresentou-se as frequências absolutas e relativas simples das variáveis qualitativas.

\section{Resultados/Conclusões}

Foram entrevistados 68 usuários-moradores, com média de idade de 43,13 anos $(\mathrm{DP}=11,7), 64,7 \%(\mathrm{n}=44)$ referiram ser do gênero masculino, 35,3\% $(\mathrm{n}=24)$ do gênero feminino, $86,8 \%(n=59)$ eram solteiros e 44,1\% $(n=30)$ referiram ser de cor da pele branca. 
Quanto ao município de origem, 27,9\% eram moradores do município de Passo Fundo $(\mathrm{n}=19), 11,8 \%$ do município Santo Antônio das Missões $(\mathrm{n}=8), 11,8 \%$ do município de Concordia $(n=8)$ e os demais de outros municípios.

O diagnóstico mais frequente foi esquizofrenia $(51,5 \%, \mathrm{n}=35)$, seguido de retardo mental grave $(8,8 \%, \mathrm{n}=6)$.

No que se refere ao estado nutricional, apenas 43 usuários-moradores estavam em condições de participar da avaliação antropométrica. Destes, 30,2\% $(n=13)$ foram classificados como eutrofia, $39,6 \%(n=17)$ como excesso de peso e 30,2\% $(n=13)$ obesidade, de acordo com o Índice de Massa Corporal (IMC).

Tais resultados corroboram com os achados de um estudo no município de Porto Alegre com usuários de um Centro de Atenção Psicossocial (CAPS), em que foi identificada pravalência de $72 \%$ de sobrepeso e obesidade (KENGERISKI et al, 2014).

Além disso, estudo realizado em Minas Gerais investigou indivíduos com transtornos mentais de residenciais terapêuticos, em uso de medicação, também foi identificada alta prevalência de sobrepeso (61\%) (GARCIA et al, 2013).

Outro estudo, realizado com 25 pacientes esquizofrênicos atendidos no Hospital de Clínicas de Porto Alegre, revelou uma frequência de 40\% de sobrepeso e 40\% de obesidade, conforme verificado pelo IMC. Entretanto estes pacientes estavam em uso de antipsicóticos (NUNES et al, 2014).

Ainda, em uma unidade de saúde em Fortaleza foi realizado um estudo que avaliou o estado nutricional de 146 indivíduos em uso de antipsicótico, sendo que 25,34\% ( $\mathrm{n}=37$ ) apresentavam sobrepeso e $28,08 \%$ ( $n=41)$ obesidade (SAMPAIO et al, 2016).

Destaca-se a elevada prevalência de algum grau de excesso de peso nessa população e em diferentes regiões do país, assim, diagnóstico nutricional pode ser coadjuvante numa intervenção adequada e eficaz no cuidado desses indivíduos.

A elevada prevalência de sobrepeso e obesidade foi identificada, no entanto, há necessidade de avaliar a influência do uso de polifarmácia, institucionalização e carências no cuidado desses indivíduos como fatores determinantes desse agravo.

\section{Nutritional status of patients with mental disorders hospitalized in therapeutic residences}

\section{Abstract}

In Brazil, rapid population aging is observed. Some populations have different characteristics of the general population, such as mortality rate, nutritional status and medication use, which is the case of individuals with mental disorders. The objective of this study was to describe the nutritional status of individuals being treated in Therapeutic Homes (RT). It is a cross-sectional study with individuals with mental disorders under treatment in RT in the city of Passo Fundo / RS. A total of 68 users were interviewed, and $39.6 \%(n=17)$ were overweight and $30.2 \%(n=13)$ were obese. Excessive use of medications appears to be a determining factor for altered nutritional status, since all subjects use at least one continuous drug and suggest that it is related to weight gain.

Keywords: Institutionalization. Mental disorders. Nutritional status. Treatment. 


\section{Referências}

FRANÇA V. V. et al. Quem são os moradores de residências terapêuticas? Perfil de usuários portadores de transtornos mentais desinstitucionalizados. Saúde Debate, Rio de Janeiro, v. 41, n. 114, p. 872-884, 2017.

GARCIA P, C, O. et al. Perfil nutricional de indivíduos com transtorno mental, usuários do Serviço Residencial Terapêutico, do município de Alfenas - MG. Revista da Universidade Vale do Rio Verde, Três Corações, v. 11, n. 1, p. 114-126, jan./jul, 2013.

GONÇALVES, S. et al. Avaliação das limitações no comportamento social em pacientes psiquiátricos de longa permanência. Ciênc. saúde coletiva, Rio de Janeiro, v.6, n.1, 2001.

JOHANSSON, L. et al. Improving nutritional status of older persons with dementia using a national preventive care program. J nutr health agin, v. 21, n. 3, 2017.

KENGERISKI, M, F. et al. Estado nutricional e hábitos alimentares de usuários em centro de atenção psicossocial de Porto Alegre, Brasil. Clin Biomed Res, v. 34, n. 3, 2014.

LEBRÃO, M. L. O envelhecimento no Brasil: Aspectos da Transição demográfica e epidemiológica. Saúde Coletiva, São Paulo, v.4, n.17, p. 135-140, Set. 2007.

LEITÃO-AZEVEDO C, L, et al. Ganho de peso e alterações metabólicas em esquizofrenia. Rev. Psiq. Clín., v. 34, n. 2, p. 184-188, 2007.

MELO, M, C, A. et al. Perfil clínico e psicossocial dos moradores em hospitais psiquiátricos no estado do Ceará, Brasil. Ciência \& Saúde Coletiva, v. 20, n.2, p. 343-352, 2015.

MINISTÉRIO DA SAÚDE. Reforma Psiquiátrica e Política de Saúde Mental no Brasil, Documento Apresentado à Conferência Regional de Reforma dos Serviços de Saúde Mental: 15 Anos Depois de Caracas. Brasília, DF: Ministério Da Saúde, 2005. Disponível em: <http://bvsms.saude.gov.br/bvs/publicacoes/ Relatorio15_anos_Caracas.pdf>. Acesso im: 28 set. 2018.

NUNES, D. et al. Estado nutricional, ingesta alimentaria y riesgo de enfermedad cardiovascular en individuos con esquizofrenia en el sur de Brasil: estudio de casos-controles. Rev Psiquiatr Salud Ment, Barcelona, v. 7, n. 2, p. 72-79, 2014.

REIS, J. S. et al. Diabetes mellitus associado com drogas antipsicóticas atípicas: relato de caso e revisão da literatura. Arquivos brasileiros de endocrinologia e Metabologia, São Paulo, v. 51, n. 3, 2007.

SAMPAIO, L. et al. Perfil socioeconômico, nutricional e fatores de risco cardiometabólico de pacientes esquizofrênicos em uso de antipsicóticos: uma reflexão para intervenção nutricional. Rev Bras Promoç Saúde, Fortaleza, v., n.1, p. 60-67. Jan./mar., 2016.

WIRSHING, D. Esquizofrenia e obesidade: impacto dos medicamentos antipsicóticos. J Clin Psychiatry, v. 65, n. 18, 2004. 